\title{
Respiratory morbidity at follow-up of small-for-gestational- age infants born very prematurely
}

\author{
Janet L. Peacock', Jessica W. Lo', Walton D'Costa², Sandra Calvert' ${ }^{3}$, Neil Marlow ${ }^{4}$ and Anne Greenough ${ }^{2}$
}

BACKGROUND: The aim of this study was to determine whether small-for-gestational-age (SGA) infants born very prematurely had increased respiratory morbidity in the neonatal period and at follow-up.

METHODS: Data were examined from infants recruited into the United Kingdom Oscillation Study (UKOS). Of the 797 infants who were born at $<29$ wk of gestational age, 174 infants were SGA. Overall, 92\% were exposed to antenatal corticosteroids and $97 \%$ received surfactant; follow-up data at 22-28 mo were available for 367 infants.

RESULTS: After adjustment for gestational age and sex, SGA infants had higher rates of supplementary oxygen dependency at 36 wk postmenstrual age (odds ratio (OR): 3.23; 95\% confidence interval: 2.03, 5.13), pulmonary hemorrhage (OR: 3.07; 95\% Cl: 1.82, 5.18), death (OR: 3.32; 95\% Cl: 2.13, 5.17), and postnatal corticosteroid requirement (OR: 2.09; 95\% Cl: 1.35, 3.23). After adjustment for infant and respiratory morbidity risk factors, a lower mean birth weight z-score was associated with a higher prevalence of respiratory admissions (OR: 1.40; 95\% Cl: $1.03,1.88$ for 1 SD change in $z$-score), cough (OR: $1.28 ; 95 \% \mathrm{Cl}$ : $1.00,1.65)$, and use of chest medicines (OR: 1.32; 95\% Cl: 1.01, 1.73).

CONCLUSION: SGA infants who were born very prematurely, despite routine use of antenatal corticosteroids and postnatal surfactant, had increased respiratory morbidity at follow-up, which was not due to poor neonatal outcome.

B etween 11 and $22 \%$ of prematurely born infants have evidence of intrauterine growth restriction (IUGR) $(1,2)$, yet the influence of fetal growth restriction on respiratory morbidity remains controversial. Some studies have highlighted that small size at birth was associated with a reduction in the incidence of respiratory distress syndrome (RDS) $(3,4)$, but other studies suggested no influence (5-9) or a significant increased risk $(10,11)$. Subsequently, there have been three large studies (12-14) that demonstrated an association between being born small for gestational age (SGA) and an increased risk of respiratory morbidity. In a study of 2,487 infants born before 37 wk of gestation, SGA infants had a significantly greater risk of developing chronic lung disease or dying and had a longer hospital stay (12). In another series, which included 2,764 very low birth weight infants born between 24 and 31 wk of gestation, SGA infants had a 3.42-fold increased risk of bronchopulmonary dysplasia (BPD) and a 4.5 -fold increased risk of death (13). In the third study, of 19,759 singleton, very low birth weight infants, born between 25 and 30 wk of gestation, IUGR was associated with increased risks of RDS and neonatal death (14). In the latter study (14), prenatal corticosteroid use was associated with decreased risks of both RDS and neonatal death. In none of the previous or the more recently reported studies (12-14), however, were infants routinely exposed to postnatal surfactant, and in the majority of studies, antenatal steroids were not routinely used. The aim of this study was to determine whether among infants born very prematurely, that is $<29$ wk of gestational age, who had been routinely exposed to both antenatal steroids and postnatal surfactant, neonatal and infant respiratory morbidity was increased in those who were born SGA.

\section{RESULTS}

Of 797 infants recruited to the United Kingdom Oscillation Study (UKOS), 22\% (174/797) were SGA infants and 205 (26\%) infants died before hospital discharge. A comparison of maternal factors by SGA status showed significant positive associations between SGA and whether the mother had preexisting hypertension, pregnancy-induced hypertension or thyroid disease, prolonged interval between membrane rupture and delivery, proven chorioamnionitis, use of tocolytic drugs, and delivery by cesarean section (Table 1). The SGA infants as compared with those who were not SGA were born at a later gestational age $(P<0.001)$. The proportion of SGA infants increased from $0 \%$ at $23 \mathrm{wk}$ of gestation to $28 \%$ at $28 \mathrm{wk}$ of gestation (Supplementary Table S1 online). None of the maternal factors showed significant associations with neonatal outcomes and so were not included in later multifactorial models in accordance with the a priori analysis plan.

SGA was associated with significantly higher rates of supplementary oxygen dependency at $36 \mathrm{wk}$ postmenstrual age (PMA) (71 vs. 53\%, $P<0.001)$ and at hospital discharge (30 vs. $19 \%, P=0.008$ ), pulmonary hemorrhage (18 vs. $11 \%$, $P=0.016$ ), and mortality (34 vs. $23 \%, P=0.004$ ). SGA infants stayed in the hospital significantly longer than those who 


\section{Articles | Peacock et al.}

Table 1. Maternal and infant characteristics by SGA status

\begin{tabular}{|c|c|c|c|c|c|}
\hline & \multicolumn{2}{|c|}{ SGA } & \multicolumn{2}{|c|}{ Not SGA } & \multirow[b]{2}{*}{$P$ value } \\
\hline & Number & Mean (SD) or $n(\%)$ & Number & Mean (SD) or $n(\%)$ & \\
\hline \multicolumn{6}{|l|}{ Maternal characteristics } \\
\hline White ethnicity & 173 & $147(85 \%)$ & 621 & $489(79 \%)$ & 0.09 \\
\hline Smoking during pregnancy & 164 & $48(29 \%)$ & 566 & $152(27 \%)$ & 0.54 \\
\hline Pregnancy-induced hypertension & 169 & $71(42 \%)$ & 608 & $49(8.1 \%)$ & $<0.001$ \\
\hline Preexisting diabetes & 174 & $3(1.7 \%)$ & 621 & $5(0.8 \%)$ & 0.30 \\
\hline Pregnancy-induced diabetes & 168 & $5(3.0 \%)$ & 604 & $6(1.0 \%)$ & 0.07 \\
\hline Hyperthyroidism or hypothyroidism & 174 & $6(3.4 \%)$ & 617 & $3(0.49 \%)$ & 0.01 \\
\hline Antenatal corticosteroids (any) & 173 & $163(94 \%)$ & 621 & $564(91 \%)$ & 0.20 \\
\hline Rupture at $<22$ wk gestation & 170 & $3(1.8 \%)$ & 614 & $23(3.7 \%)$ & 0.20 \\
\hline Chorioamnionitis & 172 & $6(3.5 \%)$ & 596 & $85(14 \%)$ & $<0.001$ \\
\hline Tocolytic drugs used & 173 & $20(12 \%)$ & 608 & $181(30 \%)$ & $<0.001$ \\
\hline \multicolumn{6}{|l|}{ Cesarean section } \\
\hline In labor & 174 & $17(9.8 \%)$ & 623 & $156(25 \%)$ & $<0.001$ \\
\hline Not in labor & 174 & $136(78 \%)$ & 623 & $144(23 \%)$ & $<0.001$ \\
\hline \multicolumn{6}{|l|}{ Infant characteristics } \\
\hline Gestational age (weeks) & 174 & $27.0(1.16)$ & 623 & $26.3(1.53)$ & $<0.001$ \\
\hline Apgar score at 5 min, mean (median) & 167 & $7.95(8)$ & 601 & $7.87(8)$ & 0.33 \\
\hline Apgar score at 10 min, mean (median) & 96 & $8.70(9)$ & 331 & $8.42(9)$ & 0.15 \\
\hline Surfactant given, yes & 174 & $170(98 \%)$ & 623 & $599(96 \%)$ & 0.33 \\
\hline \multicolumn{6}{|l|}{ Doses } \\
\hline 0 & & $4(2.3 \%)$ & & $24(3.9 \%)$ & \\
\hline 1 & & $41(24 \%)$ & & $173(28 \%)$ & \\
\hline 2 & & $98(56 \%)$ & & $333(54 \%)$ & \\
\hline 3 & & $26(15 \%)$ & & $83(13 \%)$ & \\
\hline 4 & & $5(2.9 \%)$ & & $8(1.3 \%)$ & \\
\hline 5 & & $0(0 \%)$ & & $2(0.32 \%)$ & $0.091^{b}$ \\
\hline
\end{tabular}

SGA, small for gestational age.

aBased on logistic regression with robust SEs. ${ }^{\mathrm{a}}$ Test for trend.

were not SGA (median of 107 vs. $88 \mathrm{~d}, P<0.001$ ) (Tables 2 and 3). Those effects remained significant and were greater in size after adjusting for sex and gestational age (Table 3). Regression analysis using birth weight $z$-score as the exposure variable showed a very similar picture but with stronger effects of birth weight after adjustment for gestational age and sex (Table 3).
Twenty-four month follow-up respiratory data from questionnaires completed in the prespecified window (i.e., when the children were between 22 and 28 mo of age) were available for 367 infants. Children with follow-up data tended to be sicker than those who were lost to follow-up (Supplementary Table S2 online), but the differences were not statistically significantly different. Overall, $50 \%$ of parents 
Table 2. Neonatal outcome by SGA status

\begin{tabular}{|c|c|c|c|c|c|}
\hline & \multicolumn{2}{|c|}{ SGA } & \multicolumn{2}{|c|}{ Not SGA } & \multirow[b]{2}{*}{$P$ value } \\
\hline & $N$ & Mean (SD) or $n(\%)$ & $N$ & Mean (SD) or $n(\%)$ & \\
\hline Death/oxygen dependency at $36 \mathrm{wk}$ & 174 & $139(80 \%)$ & 623 & $394(63 \%)$ & $<0.001$ \\
\hline$<7 d$ & 60 & $33(55 \%)$ & 145 & $77(53 \%)$ & \\
\hline$<28 \mathrm{~d}$ and $\geq 7 \mathrm{~d}$ & 60 & $14(23 \%)$ & 145 & $46(32 \%)$ & \\
\hline Oxygen dependency at $36 \mathrm{wk}$ & 119 & $84(71 \%)$ & 489 & $259(53 \%)$ & 0.001 \\
\hline Survival without oxygen dependency at $36 \mathrm{wk}$ & 114 & $35(31 \%)$ & 478 & $229(48 \%)$ & \\
\hline Home oxygen at discharge & 113 & $34(30 \%)$ & 475 & $88(19 \%)$ & 0.006 \\
\hline Age of infant (weeks) & 34 & $19.4(6.9)$ & 87 & $17.4(6.0)$ & 0.12 \\
\hline Hospital stays mean in days (median) & 114 & $114(107)$ & 475 & $94(88)$ & $<0.001$ \\
\hline
\end{tabular}

SGA, small for gestational age.

Table 3. The relationship between neonatal outcomes and being small for gestational age, adjusted for birth factors

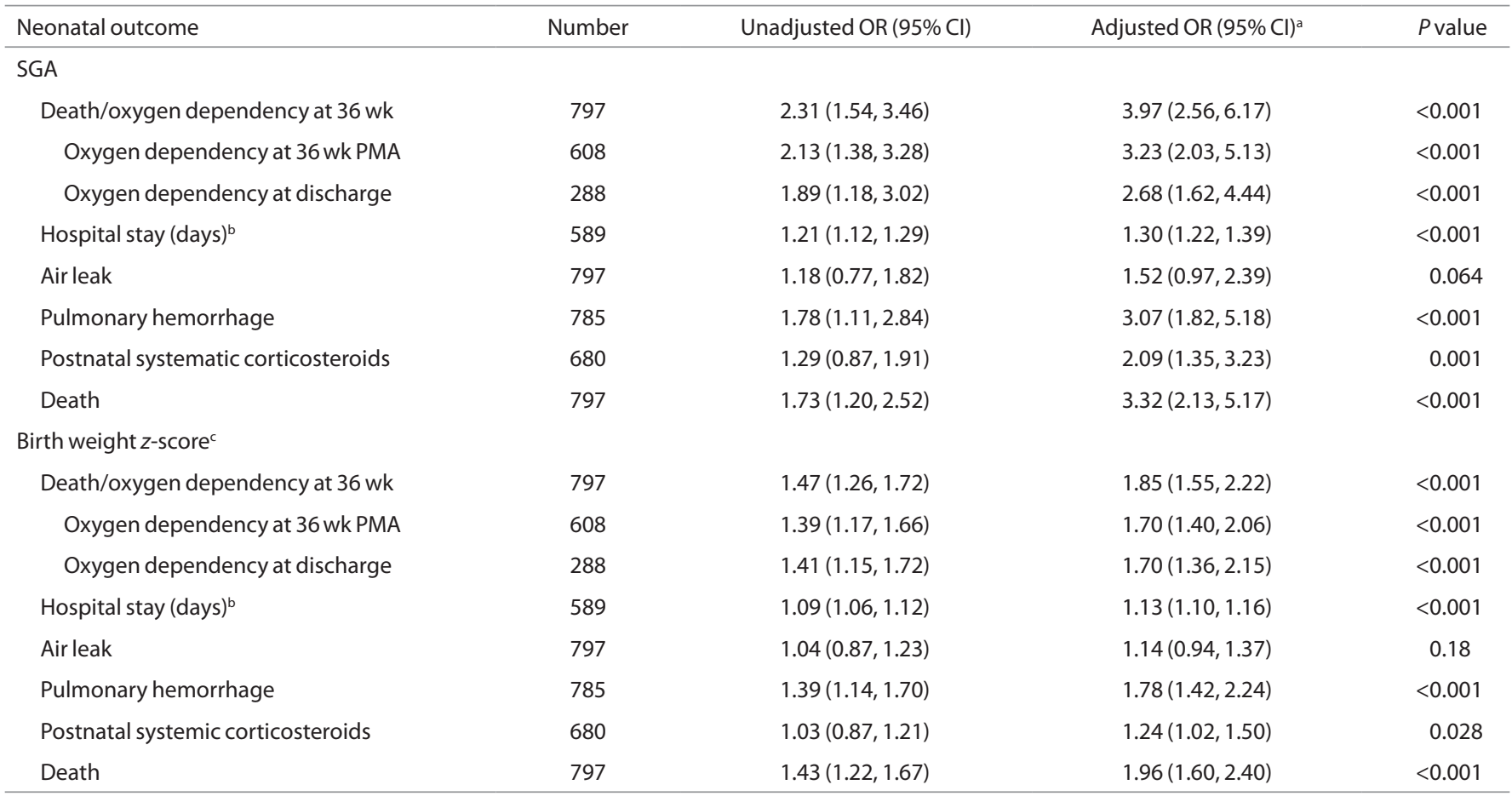

$\mathrm{Cl}$, confidence interval; PMA, postmenstrual age; SGA, small for gestational age.

adjusted for sex and gestational age. 'oog transformed. 'Results are presented as odds ratios (ORs) equivalent to a 1 SD decrease in birth weight $z$-score.

reported that their children had had cough and $58 \%$ had received chest medicines (Table 4). A greater proportion of SGA infants required hospital respiratory readmissions (30 vs. $19 \%, P=0.035$ ), but there were no significant associations of SGA and follow-up respiratory outcomes after adjustments (Table 5). In addition, there was no significant correlation between a diagnosis of BPD and longer-term respiratory outcomes. Birth weight $z$-score was negatively associated with respiratory hospital admissions and use of chest medicines. Such associations remained significant and became more marked after adjusting for sex and gestational age (Table 5).
After further adjustment for oxygen dependency at $36 \mathrm{wk}$ PMA, the relationships among birth weight $z$-score, respiratory hospital admission, and use of chest medicines remained significant. Final adjustment for sex, gestational age, oxygen dependency at 36 wk PMA, living with siblings younger than $5 \mathrm{y}$ of age, having a twin/triplet, and living with a mother or father who smoked showed that five adverse respiratory outcomes (respiratory hospital admission, cough, use of any chest medicine, use of bronchodilators or inhaled steroids, and use of antibiotics) at follow-up were negatively and significantly associated with birth weight $z$-score. 


\section{Articles | Peacock et al.}

Table 4. Respiratory outcomes at 24-mo follow-up by SGA status

\begin{tabular}{|c|c|c|c|c|c|}
\hline & \multicolumn{2}{|c|}{ SGA } & \multicolumn{2}{|c|}{ Not SGA } & \multirow[t]{2}{*}{$P$ value ${ }^{\mathrm{a}}$} \\
\hline & Number & $n(\%)$ & Number & $n(\%)$ & \\
\hline Hospital respiratory admission & 77 & $23(30 \%)$ & 285 & $53(19 \%)$ & 0.035 \\
\hline Wheeze & 76 & $29(38 \%)$ & 278 & $102(37 \%)$ & 0.82 \\
\hline Chest medicines (all types) & 77 & $47(61 \%)$ & 286 & $162(57 \%)$ & 0.50 \\
\hline Antibiotics & 77 & $33(43 \%)$ & 287 & $101(35 \%)$ & 0.23 \\
\hline
\end{tabular}

SGA, small for gestational age.

abed on logistic regression with robust SES.

Table 5. Relationships between being small for gestational age and respiratory outcomes at 24 mo

\begin{tabular}{|c|c|c|c|c|c|c|}
\hline Respiratory illness at $24 \mathrm{mo}$ & Number $^{\mathrm{a}}$ & $\begin{array}{c}\text { Unadjusted OR } \\
\quad(95 \% \mathrm{Cl})\end{array}$ & $\begin{array}{l}\text { Adjusted OR } \\
(95 \% \mathrm{Cl})^{\mathrm{b}}\end{array}$ & $\begin{array}{l}\text { Adjusted OR } \\
(95 \% \mathrm{CI})^{c}\end{array}$ & $\begin{array}{l}\text { Adjusted OR } \\
(95 \% \mathrm{Cl})^{\mathrm{d}}\end{array}$ & $P$ value \\
\hline \multicolumn{7}{|l|}{ SGA } \\
\hline Cough & 366 & $1.41(0.84,2.34)$ & $1.49(0.89,2.51)$ & $1.43(0.84,2.43)$ & $1.31(0.74,2.32)$ & 0.35 \\
\hline Wheeze & 354 & $1.06(0.62,1.81)$ & $1.18(0.68,2.05)$ & $1.16(0.66,2.06)$ & $1.15(0.62,2.13)$ & 0.67 \\
\hline Antibiotics & 364 & $1.38(0.82,2.33)$ & $1.46(0.85,2.51)$ & $1.14(0.70,1.86)$ & $1.37(0.74,2.51)$ & 0.32 \\
\hline \multicolumn{7}{|l|}{ Birth weight $z$-score ${ }^{f}$} \\
\hline Respiratory hospital admission & 362 & $1.43(1.12,1.81)$ & $1.59(1.22,2.06)$ & $1.51(1.16,1.98)$ & $1.40(1.03,1.88)$ & 0.032 \\
\hline Cough & 366 & $1.24(0.99,1.53)$ & $1.27(1.02,1.59)$ & $1.26(1.00,1.58)$ & $1.28(1.00,1.65)$ & 0.049 \\
\hline Antibiotics & 364 & $1.24(1.00,1.54)$ & $1.29(1.03,1.62)$ & $1.29(1.02,1.63)$ & $1.38(1.06,1.80)$ & 0.018 \\
\hline
\end{tabular}

$\mathrm{Cl}$, confidence interval; $\mathrm{PMA}$, postmenstrual age; $\mathrm{OR}$, odds ratio; SGA, small for gestational age.

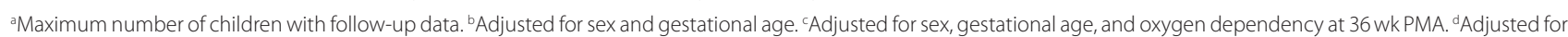

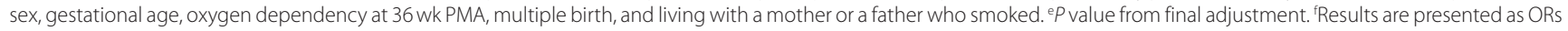
equivalent to a 1 SD decrease in birth weight z-score.

We performed further analyses using oxygen dependency at $28 \mathrm{~d}$ rather than oxygen dependency at $36 \mathrm{wk}$ PMA to see if this affected the findings; the results were not appreciably different and so are not reported here.

\section{DISCUSSION}

We have demonstrated that SGA, very prematurely born infants, despite routine exposure to antenatal steroids and postnatal surfactant, had increased respiratory morbidity in the neonatal period and at follow-up as compared with those who were not SGA. The associations remained significant after adjusting for infant factors and possible risk factors for chronic respiratory morbidity. As compared with using a cutoff to define abnormal size at birth, we showed even stronger relationships between adverse respiratory outcomes and birth weight $z$-scores, which would indicate that poor growth in utero adversely affects neonatal and infant respiratory outcomes. It has been suggested that neonatal growth standards based on healthy populations could improve the identification of very preterm neonates as SGA and at risk of intraventricular hemorrhage (1). In this study, although we found a number of maternal diseases to be associated with being SGA, none of those factors showed significant associations with neonatal outcomes and hence were not included in later models. Our results are in line with those of another study (15), which showed that among a very preterm cohort stratifying for pregnancy complications yielded similar risk patterns; that is, BPD declined continuously with increasing birth weight. Our finding of stronger relationships using birth weight $z$-score rather than the binary outcome, above or below the 10th percentile, suggests that the 10th percentile is not a true threshold and that growth should be analyzed as a continuum. A threshold, however, is useful clinically and so it is recommended that analyses include both the binary and continuous outcomes to provide a complete picture.

The mortality rate in the UKOS overall was $26 \%$. Infants born at $25 \mathrm{wk}$ plus $6 \mathrm{~d}$ or less had a mortality rate of $42 \%$, 
which is comparable with the $39 \%$ mortality rate of infants in the EPICURE study, who were all born at $25 \mathrm{wk}$ of gestation or less but in 1995 (16). In a prospective cohort study in a geographically defined population in the UK during 2000-2005, the survival rate to discharge of infants born at $<25+6$ wk was $47 \%$ (17). By contrast to previous studies, the majority ( $>90 \%$ of infants) were routinely exposed to antenatal steroids and postnatal surfactant. In one recently reported study (2) among infants with a birth weight $<1,000 \mathrm{~g}$ and exposed to antenatal steroids and postnatal surfactant, those with SGA had a lower incidence of RDS and BPD. Antenatal steroid use, however, was $<80 \%$; it is not clear how many infants received surfactant, and infants up to $34 \mathrm{wk}$ were included in the analysis. Our results demonstrate that neither antenatal steroid nor postnatal surfactant exposure mitigated the adverse influence of IUGR on neonatal and infant respiratory morbidity. Those findings are in accordance with the results of randomized controlled trials demonstrating that neither treatment reduces $\operatorname{BPD}(18,19)$.

We did not find a significant correlation between a diagnosis of BPD and longer-term, adverse respiratory outcomes. BPD, in this study, was diagnosed in infants who were receiving supplementary oxygen beyond 36 wk postmenstrual age. No oxygen reduction test was undertaken (20). Such a definition has previously been shown to correlate poorly with longer-term respiratory outcomes (21). Diagnosing infants as having BPD because they are supplementary oxygen dependent at $28 \mathrm{~d}$ and then assessing BPD severity at 36 wk PMA (22) has been shown to correlate much better with longer-term outcomes (23).

The lack of effect of antenatal glucocorticoids on BPD development has been ascribed to increased survival of very immature infants, but an alternative hypothesis is that antenatal steroids are the first hit taken by the fetal lung, which primes the lung for more ventilation-induced injury (24). Antenatal glucocorticoid treatment is given to women at risk of preterm delivery because it decreases the risk of death, RDS, and intraventricular hemorrhage (25). Preterm rupture of the membranes and histological chorioamnionitis are not contraindications to maternal glucocorticoids $(26,27)$, and women with undiagnosed chorioamnionitis with preterm labor currently receive corticosteroids to enhance fetal lung maturation $(26,27)$. In a subgroup of preterm infants, however, it has been suggested maternal glucorticoids may increase lung inflammation, which could promote the subsequent development of BPD (28).

There are possible limitations to our study; these include that 2 -y follow-up data were not available for all survivors. The children who were followed up tended to be sicker as compared with those who were not, but the differences did not reach statistical significance. The choice of growth standards affects the cutoff to define an SGA infant. We used British charts from 1990 (29) as also used in many other studies, including the EPICURE study (16). More recent standards produced from the United States (30) are based on larger numbers, but their ethnic mix is different from that in the UK study and so they were not suitable. The infants included in the current study had been entered into a randomized trial examining the efficacy of high-frequency oscillation and conventional mechanical ventilation as modes of respiratory support for very prematurely born infants (31). We have, however, previously shown that both short-term (31) and 2-y follow-up (32) outcomes were similar in the two groups. Respiratory follow-up data at 22-28 mo was collected during a pediatrician consultation; therefore, it is possible that there may have been faulty parental recall. However, this would have similarly affected parents of children with or without SGA. In addition, the analysis of the results of the contemporaneously collected neonatal data yielded similar findings to the analysis of the follow-up data, that is, increased respiratory morbidity in the children who had been born SGA.

Our results demonstrated among infants born very prematurely that those who were SGA had greater longer-term respiratory morbidity, and these results are predictable from those obtained from studies on animal models. In a series of studies of respiratory development in growth-restricted ovine fetuses, Harding et al. (33) demonstrated that the lungs of growthrestricted fetuses were structurally immature and their tracheal and bronchial development was impaired. Other studies have demonstrated that normal lung development is critically dependent on the presence of appropriate oxygen tensions and nutrition (34), which are reduced in SGA fetuses. In both sheep (35) and rats (36), the reduced number of alveoli associated with IUGR at birth persisted into adulthood with no evidence of catch-up growth. Those data are in line with the finding that lung function at school age was reduced in prematurely born infants who had experienced IUGR as compared with genderand age-matched children born at term (37).

In conclusion, we have demonstrated that being born SGA, despite routine exposure of antenatal steroids and postnatal surfactant, is associated with increased neonatal and infant respiratory morbidity, which was not explained by other risk factors. Our results emphasize the importance of investigating therapies to prevent fetal growth restriction and improving the postnatal management of affected infants to improve their long-term outcome.

\section{METHODS}

Data were examined from infants recruited into the UKOS, a multicenter, randomized controlled trial conducted between August 1998 and January 2001 (31). Infants were randomized to either highfrequency oscillation or conventional mechanical ventilation within $1 \mathrm{~h}$ of birth. The study was approved by the South Thames Multicentre Research Ethics Committee and the local research ethics committee at each participating center. Parents gave informed written consent for the infant to take part in the randomized trial and the follow-up.

Data were available for the mother's age, ethnicity, smoking during pregnancy, preexisting or pregnancy-induced hypertension requiring treatment, preexisting or pregnancy-induced diabetes requiring treatment, thyroid disease, use of antenatal corticosteroids taken before birth, whether an antepartum hemorrhage or rupture of membranes had occurred, use of tocolytic drugs, and mode of delivery. Infant factors obtained from the database included birth weight; sex; gestational age; multiple birth; Apgar scores at 1, 5, and $10 \mathrm{~min}$; and the use of surfactant. Infants were diagnosed as having been born SGA if their birth weight was $<10$ th percentile (i.e., birth weight $z$-score $\leq-1.28$ ). The birth weight $z$-score (i.e., SD score) was calculated for each infant using the British 1990 reference (29).

Neonatal outcomes were BPD, that is, supplementary oxygen dependency at 36 wk PMA and hospital discharge; an oxygen reduction test was not undertaken (20). A secondary outcome (see sensitivity 
analysis) was oxygen dependency at $28 \mathrm{~d}$. Other outcomes were air leak, pulmonary hemorrhage, death, and use of postnatal corticosteroids. The infants were followed up to a corrected age of $24 \mathrm{mo}$, when pediatricians completed questionnaires by asking parents about their infant's respiratory health and risk factors for respiratory morbidity. The pediatricians were asked to complete the questionnaire within a prespecified window, that is, when the children were between 22 and 28 mo of age. The questions asked were as follows: whether the child suffered from cough and/or wheeze in the past $12 \mathrm{mo}$; and whether the child had had hospital admission(s) for respiratory problems and/ or had received any medications (bronchodilators, inhaled steroids, or antibiotics) for respiratory disorders.; Questions were also asked about risk factors for respiratory morbidity, that is whether the child lived with long-haired pets or in a rented accommodation; if there had been multiple births; whether the infant lived with a mother or father who smoked or had contact with smokers for $>15 \mathrm{~h}$ per wk; or whether the infant lived with siblings who were $<5$ y of age. Follow-up respiratory outcomes were respiratory hospital admissions and use of chest medicines (bronchodilators, inhaled steroids, and/or antibiotics).

\section{Analysis}

The analyses were performed using both the dichotomized SGA (yes/ no) and the birth weight $z$-score because the latter analysis gives greater statistical power (38). A statistical analysis plan was established a priori to avoid ad hoc modeling decisions: we first explored maternal factors as well as which birth factors affected intrauterine growth, and then, after adjusting for those, which neonatal outcomes were influenced by SGA. Analysis was then undertaken to determine any effects of SGA on respiratory morbidity after adjusting, in three stages, for all confounding maternal and infant factors, neonatal outcomes, and sociodemographic risk factors. The choice of factors for adjustment was guided by previous analyses of the UKOS data (39).

Unifactorial analyses were initially undertaken, and variables significant at $P<0.10$ were then modeled in multifactorial regressions. Logistic regression or linear regression was used to examine associations for binary or continuous outcomes, respectively. Odds ratios for SGA/not SGA and the odds ratio equivalent to 1 SD decrease in birth weight $z$-score are reported. Skewed continuous data were log-transformed as appropriate. Robust SEs were used to take into account the clustering among multiple births (40). All analyses were conducted using Stata v 12.1 (2012; Statacorp, College Station, TX).

\section{SUPPLEMENTARY MATERIAL}

Supplementary material is linked to the online version of the paper at http://www.nature.com/pr

\section{STATEMENT OF FINANCIAL SUPPORT}

J.W.L. was supported by a National Institute for Health Research/Health Technology Assessment grant and the National Institute for Health Research Biomedical Research Centre at Guy's and St Thomas' Hospital/ King's College London. A.G. and N.M. are National Institute for Health Research Senior Investigators. The United Kingdom Oscillation Study was funded by the Medical Research Council, and N.M. receives partial funding from the Department of Health's National Institute for Health Research Biomedical Research Centre's funding scheme at University College London Hospitals/University College London.

\section{REFERENCES}

1. Ferdynus C, Quantin C, Abrahamowicz M, et al. Can birth weight standards based on healthy populations improve the identification of small-forgestational-age newborns at risk of adverse neonatal outcomes? Pediatrics 2009;123:723-30.

2. Yu HJ, Kim ES, Kim JK, et al. Outcomes of small for gestational age micropremies depending on how young or how small they are. Korean J Pediatr 2011;54:246-52.

3. Gluck L, Kulovich MV. Lecithin-sphingomyelin ratios in amniotic fluid in normal and abnormal pregnancy. Am J Obstet Gynecol 1973;115:539-46.

4. Procianoy RS, Garcia-Prats JA, Adams JM, Silvers A, Rudolph AJ. Hyaline membrane disease and intraventricular haemorrhage in small for gestational age infants. Arch Dis Child 1980;55:502-5.
5. Piper JM, Langer O. Is lung maturation related to fetal growth in diabetic or hypertensive pregnancies? Eur J Obstet Gynecol Reprod Biol 1993;51:15-9.

6. Ruys-Dudok van Heel I, de Leeuw R. Clinical outcome of small for gestational age preterm infants. J Perinat Med 1989;17:77-83.

7. Thompson PJ, Greenough A, Gamsu HR, Nicolaides KH. Ventilatory requirements for respiratory distress syndrome in small-for-gestationalage infants. Eur J Pediatr 1992;151:528-31.

8. Henderson-Smart DJ. Postnatal consequences of chronic intrauterine compromise. Reprod Fertil Dev 1995;7:559-65.

9. Piper JM, Xenakis EM, McFarland M, Elliott BD, Berkus MD, Langer O. Do growth-retarded premature infants have different rates of perinatal morbidity and mortality than appropriately grown premature infants? Obstet Gynecol 1996;87:169-74.

10. Fleischer A, Anyaegbunam A, Guidetti D, Randolph G, Merkatz IR. A persistent clinical problem: profile of the term infant with significant respiratory complications. Obstet Gynecol 1992;79:185-90.

11. Tyson JE, Kennedy K, Broyles S, Rosenfeld CR. The small for gestational age infant: accelerated or delayed pulmonary maturation? Increased or decreased survival? Pediatrics 1995;95:534-8.

12. Sharma P, McKay K, Rosenkrantz TS, Hussain N. Comparisons of mortality and pre-discharge respiratory outcomes in small-for-gestational-age and appropriate-for-gestational-age premature infants. BMC Pediatr 2004;4:9.

13. Regev RH, Lusky A, Dolfin T, Litmanovitz I, Arnon S, Reichman B; Israel Neonatal Network. Excess mortality and morbidity among small-forgestational-age premature infants: a population-based study. J Pediatr 2003;143:186-91.

14. Bernstein IM, Horbar JD, Badger GJ, Ohlsson A, Golan A. Morbidity and mortality among very-low-birth-weight neonates with intrauterine growth restriction. The Vermont Oxford Network. Am J Obstet Gynecol 2000;182(1 Pt 1):198-206.

15. Zeitlin J, El Ayoubi M, Jarreau PH, et al.; MOSAIC Research Group. Impact of fetal growth restriction on mortality and morbidity in a very preterm birth cohort. J Pediatr 2010;157:733-9.e1.

16. Costeloe K, Hennessy E, Gibson AT, Marlow N, Wilkinson AR. The EPICure study: outcomes to discharge from hospital for infants born at the threshold of viability. Pediatrics 2000;106:659-71.

17. Field DJ, Dorling JS, Manktelow BN, Draper ES. Survival of extremely premature babies in a geographically defined population: prospective cohort study of 1994-9 compared with 2000-5. BMJ 2008;336:1221-3.

18. Stewart A, Brion LP, Soll R. Diuretics for respiratory distress syndrome in preterm infants. Cochrane Database Syst Rev 2011:CD001454.

19. Shah SS, Ohlsson A, Halliday H, Shah VS. Inhaled versus systemic corticosteroids for the treatment of chronic lung disease in ventilated very low birth weight preterm infants. Cochrane Database Syst Rev 2007:CD002057.

20. Walsh MC, Yao Q, Gettner P, et al.; National Institute of Child Health and Human Development Neonatal Research Network. Impact of a physiologic definition on bronchopulmonary dysplasia rates. Pediatrics 2004;114:1305-11.

21. Kinali M, Greenough A, Dimitriou G, Yüksel B, Hooper R. Chronic respiratory morbidity following premature delivery-prediction by prolonged respiratory support requirement? Eur J Pediatr 1999;158:493-6.

22. Jobe AH, Bancalari E. Bronchopulmonary dysplasia. Am J Respir Crit Care Med 2001;163:1723-9.

23. Ehrenkranz RA, Walsh MC, Vohr BR, et al.; National Institutes of Child Health and Human Development Neonatal Research Network. Validation of the National Institutes of Health consensus definition of bronchopulmonary dysplasia. Pediatrics 2005;116:1353-60.

24. Jobe AH. Antenatal factors and the development of bronchopulmonary dysplasia. Semin Neonatol 2003;8:9-17.

25. Crowley P. Antenatal corticosteroid therapy: a metanalysis of the randomized trials 1972-1994. Am J Obstet Gynecol 1995;173:322-35.

26. Elimian A, Verma U, Beneck D, et al. Histologic chorioamnionitis, antenatal steroids and perinatal outcomes. Obstet Gynecol 2000;96:333-6.

27. Harding JE, Pang J, Knight DB, Liggins GC. Do antenatal corticosteroids help in the setting of preterm rupture of membranes? Am J Obstet Gynecol 2001;184:131-9. 
28. Kallapur SG, Kramer BW, Moss TJ, et al. Maternal glucocorticoids increase endotoxin-induced lung inflammation in preterm lambs. Am J Physiol Lung Cell Mol Physiol 2003;284:L633-42.

29. Cole TJ, Freeman JV, Preece MA. British 1990 growth reference centiles for weight, height, body mass index and head circumference fitted by maximum penalized likelihood. Stat Med 1998;17:407-29.

30. Olsen IE, Groveman SA, Lawson ML, Clark RH, Zemel BS. New intrauterine growth curves based on United States data. Pediatrics 2010;125:e214-24.

31. Johnson AH, Peacock JL, Greenough A, et al.; United Kingdom Oscillation Study Group. High frequency oscillatory ventilation for the prevention of chronic lung disease of prematurity. N Engl J Med 2002;347:633-42.

32. Marlow N, Greenough A, Peacock JL, et al. Randomised trial of high frequency oscillatory ventilation or conventional ventilation in babies of gestational age 28 weeks or less: respiratory and neurological outcomes at 2 years. Arch Dis Child Fetal Neonatal Ed 2006;91:F320-6.

33. Harding R, Cock ML, Louey S, et al. The compromised intra-uterine environment: implications for future lung health. Clin Exp Pharmacol Physiol 2000;27:965-74.
34. Maritz GS, Morley CJ, Harding R. Early developmental origins of impaired lung structure and function. Early Hum Dev 2005;81:763-71.

35. Maritz GS, Cock ML, Louey S, Suzuki K, Harding R. Fetal growth restriction has long-term effects on postnatal lung structure in sheep. Pediatr Res 2004;55:287-95

36. Karadag A, Sakurai R, Wang Y, et al. Effect of maternal food restriction on fetal rat lung lipid differentiation program. Pediatr Pulmonol 2009;44:635-44.

37. Morsing E, Gustafsson P, Brodszki J. Lung function in children born after foetal growth restriction and very preterm birth. Acta Paediatr 2012;101: 48-54.

38. Altman DG, Royston P. The cost of dichotomising continuous variables. BMJ 2006;332:1080.

39. Peacock JL, Marston L, Marlow N, Calvert SA, Greenough A. Neonatal and infant outcome in boys and girls born very prematurely. Pediatr Res 2012;71:305-10

40. Marston L, Peacock JL, Yu K, et al. Comparing methods of analysing datasets with small clusters - case studies using four paediatric datasets. Paediatr Perinatal Epidemiol 2009;23:380-92. 